\title{
Pharmacological Inhibition of Phosholipase A2: Results from Phase 3 Clinical Trials with Darapladib and Varespladib in Patients with Cardiovascular Disease
}

\author{
Nicola Ferri ${ }^{1,2^{*}}$, Chiara Ricci ${ }^{1}$ and Alberto Corsini ${ }^{1,2}$ \\ ${ }^{1}$ Dipartimento di Scienze Farmacologiche e Biomolecolari, Università degli Studi di Milano, Italy \\ ${ }^{2}$ IRCCS, Multimedia, Milan Italy
}

\begin{abstract}
The hydrolysis of the ester bond of glycerophospholipids is catalyzed by the family of enzymes Phospholipase A2 $\left(P L A_{2}\right)$, that leads to a release of free fatty acids and lysophospholipids, including the arachidonic acid, the precursor of the eicosanoids and the inflammatory cascades. The mass and the enzymatic activity of PLA, have been positively correlated with the incidence of cardiovascular diseases in epidemiological and genetic studies. In particular, several experimental evidences have shown that PLA, identified in the atherosclerotic plaque, are directly involved in the proatherogenic inflammatory response. From these evidences, $\mathrm{PLA}_{2}$ have become a potential pharmacological target of considerable interest and two different PLA inhibitors have been developed: varespladib, a reversible $S P L A_{2}$ inhibitor, and darapladib, a selective $L p-P L A_{2}$ inhibitor. Both these two small molecules have been tested both on animal models, where they have shown anti-atherosclerotic properties, and in phase 2 clinical trials, where they have demonstrated positive effects on atherosclerotic plaque composition. Unfortunately, the following three phase 3 trials, which have been recently published, did not shown any additional protective action of PLA inhibitors neither in co-administration with statins and antiplatelet drugs, nor in coronary revascularization. In the first one, the VISTA-16 study, varespladib has been administrated to patients with acute coronary syndrome, in the second and third one, the Stability and the SOLID-TIMI 52 studies, darapladib has been administrated to patients with stable coronary heart disease and acute coronary syndrome, respectively. The present article is focused on the enzymatic properties and on the involvement of sPLA ${ }_{2}$ and Lp-PLA ${ }_{2}$ in atherogenesis, with particular attention on the results of experimental and clinical studies with both varespladib and darapladib.
\end{abstract}

Keywords: Darapladib; Cardiovascular diseases; Atherosclerosis; Varespladib

\section{Introduction}

In the recent years, it has been documented a significant reduction in morbidity and mortality from cardiovascular causes. Nevertheless, it is evident that even in patients treated aggressively with currently available drugs; the rate of cardiovascular events still remains high. These conditions highlight the need to develop new strategies for the treatment of cardiovascular disease. In the mid 90, several evidences have support the hypothesis for the key role of vascular inflammation in atherogenesis, as documented by the involvement of pro-inflammatory molecules, such as C-reactive protein (CRP), interleukin-1 (IL-1), p38 MAPK and phospholipase $\mathrm{A}_{2}$ both soluble $\left(\mathrm{sPLA}_{2}\right)$ or associated with lipoproteins (Lp-PLA $A_{2}$ ). The role of inflammatory response in atherogenesis has also been confirmed by the evidence of the so called "pleiotropic" effects of statins (the most potent antiatherosclerotic agents currently available in clinic) that could affect the vascular response to injury and reduces the inflammatory marker CRP [1-8]. The Lp-PLA2, in particular, has been considered to be a potential pharmacological target for the development of new drugs with antiatherosclerotic activity [9].

\section{Role of sPLA and Lp-PLA ${ }_{2}$ in the Atherosclerosis}

sPLA $_{2}$

The PLA represent a class of enzymes that hydrolyze the sn- 2 ester bond of glycerophospholipids leading to the formation of free fatty acids and lysophospholipids, such as arachidonic acid, the precursor of the eicosanoids. The soluble PLA are divided into 10 groups, which include 13 different isoforms; in particular, the sPLA $\mathrm{A}_{2}$-IIA, the sPLA III, the $\mathrm{PPLA}_{2}-\mathrm{V}$ and the $\mathrm{PLA}_{2}-\mathrm{X}$ are involved in atherogenesis(Table
1) $[10,11]$. The four isoforms show a different capacity to hydrolyze the phosphatidylcholine (PC) and phosphatidylethanolamine (PE) and the $\mathrm{SPLA}_{2}-\mathrm{V}$ has a unique role in hydrolyzing phospholipids present in human lipoproteins [12-14]. All these four isoforms of soluble PLA are $_{2}$ present in the atherosclerotic plaques [15,16], although with a different distribution. The $\mathrm{SPLA}_{2}-\mathrm{V}$ is mainly expressed in smooth muscle cells while the other (IIA, III, and X) are also present in macrophages [13]. Regarding the role of $\mathrm{sLA}_{2}$ in atherogenesis, it is generally accepted, that these enzymes are capable to modify the LDL and increase the ability to bind to proteoglycans of the extracellular matrix present in the vessel wall facilitating their aggregation and oxidation. The enzymatic activity of both sPLA and Lp-PLA leads to the formation of bioactive fatty acids (such as arachidonic acid) and lysophosphatidylcholine (lyso-PC) capable to promote cell activation and the production of pro-inflammatory cytokines. The sPLA also promotes the formation of macrophage-derived foam cells by modifying the lipoprotein particles.

\section{Lp-PLA}

Differently from the sPLA, there is only one isoform of Lp-PLA

*Corresponding author: Nicola Ferri, Dipartimento di Scienze Farmacologiche e Biomolecolari, Università degli Studi di Milano, Italy, Via Balzaretti 9, 20133 Milano, Tel: 0250318322; E-mail: nicola.ferri@unimi.it

Received: March 31, 2015; Accepted: April 20, 2015; Published: April 26, 2015

Citation: Ferri N, Ricci C, Corsini A (2015) Pharmacological Inhibition of Phosholipase A2: Results from Phase 3 Clinical Trials with Darapladib and Varespladib in Patients with Cardiovascular Disease. Cardiol Pharmacol 4: 137. doi:10.4172/2329-6607.1000137

Copyright: (c) 2015 Ferri $\mathrm{N}$ et al . This is an open-access article distributed under the terms of the Creative Commons Attribution License, which permits unrestricted use, distribution, and reproduction in any medium, provided the original author and source are credited. 
Citation: Ferri N, Ricci C, Corsini A (2015) Pharmacological Inhibition of Phosholipase A2: Results from Phase 3 Clinical Trials with Darapladib and Varespladib in Patients with Cardiovascular Disease. Cardiol Pharmacol 4: 137. doi:10.4172/2329-6607.1000137

Page 2 of 5

\begin{tabular}{|c|c|c|c|c|c|c|c|c|}
\hline & \multicolumn{2}{|c|}{ Structural features } & \multicolumn{6}{|c|}{ Enzymatic properties } \\
\hline & $\begin{array}{l}\text { Molecular } \\
\text { Mass }(\mathrm{kDa})\end{array}$ & $\begin{array}{l}\text { Active } \\
\text { Site }\end{array}$ & $\begin{array}{l}\mathrm{PE} \\
\text { Hydrolysis }\end{array}$ & $\begin{array}{l}\text { PC } \\
\text { Hydrolysis }\end{array}$ & $\begin{array}{l}\text { PAF } \\
\text { Hydrolysis }\end{array}$ & $\begin{array}{l}\text { oxPL } \\
\text { Hydrolysis }\end{array}$ & $\begin{array}{l}\text { P r o t e o g I y c a } n \\
\text { Binding }\end{array}$ & $\begin{array}{l}\text { Foam } \\
\text { Cell Formation }\end{array}$ \\
\hline sPLA ${ }_{2}-I I A$ & 13.9 & His/Asp & +++ & $+/-$ & - & $? \dagger$ & +++ & + \\
\hline sPLA ${ }_{2}-I I I$ & 18.3 & His/Asp & ++ & ++ & - & $? \dagger$ & - & ++ \\
\hline SPLA $-V$ & 13.8 & His/Asp & +++ & +++ & - & $? \dagger$ & ++ & ++ \\
\hline SPLA $-X$ & 13.6 & His/Asp & +++ & +++ & ++ & $? \dagger$ & - & ++ \\
\hline Lp-PLA & 45 & Ser/Asp/His & - & - & +++ & +++ & - & - \\
\hline
\end{tabular}

PE=Phosphatidylethanolamine; PC=Phosphatidylcholine; $\mathrm{SPLA}_{2} \mathrm{~s}$ and Lp-PLA have different molecular masses and different active sites: a His/Asp dyad and a Ser/Asp/ His triad, respectively.

Table 1: Structural and functional features of $s P L A_{2}$ and $L p-P L A_{2}$

\begin{tabular}{|c|c|}
\hline Darapladib & Pharmacological properties \\
\hline & Inhibition of Lp-PLA; $; \mathrm{IC}_{50}=0.25 \mathrm{nM}[28]$ \\
\hline & $43 \%, 55 \%$, and $66 \%$ inhibition at the doses of 40,80 e $160 \mathrm{mg}$ of $L p-P L A_{2}$, respectively, in clinical trials [37] \\
\hline & Experimental models \\
\hline & Reduction of Lp-PLA 2 activity in experimental and human atherosclerotic lesions [34] \\
\hline & Phase 2 trials \\
\hline & Reduction of Lp-PLA ${ }_{2}$ activity in carotid plaques [35] \\
\hline & $\begin{array}{l}\text { Inhibition of the progression of the necrotic core volume of coronary plaques in the IBIS-2 trial conducted in patients with } \\
\text { cardiovascular disease [33] }\end{array}$ \\
\hline & Phase 3 trials \\
\hline & $\begin{array}{l}\text { No reduction of the primary endpoint of cardiovascular death, myocardial infarction or stroke in the STABILITY trial in patients with } \\
\text { stable coronary artery disease. Significant reduction in the secondary endpoint of total and major coronary events [39] }\end{array}$ \\
\hline & $\begin{array}{l}\text { No reduction of the primary endpoint of cardiovascular death, myocardial infarction and revascularization in the SOLID-TIMI } 52 \\
\text { trial in patients with acute coronary syndrome. No positive effect on the secondary endpoint of the study [40]. }\end{array}$ \\
\hline \multirow{11}{*}{ Varespladib } & Pharmacodynamic properties \\
\hline & Inhibition of $\mathrm{SPLA}_{2}-\| \mathrm{A} ; \mathrm{IC}_{50}=6.2 \mathrm{nM}[30]$; At similar concentrations it also inhibits sPLA $-V$ e $X[29]$ \\
\hline & $84 \%$ inhibition of sPLA ${ }_{2}-I I A$ at a dose of $500 \mathrm{mg}$ in clinical trials [29] \\
\hline & Experimental models \\
\hline & Reduction of atherosclerotic plaque area and total cholesterol levels in mouse models of atherosclerosis[31,32] \\
\hline & Attenuation of the development of aneurysm induced by angiotensin II [31] \\
\hline & Phase 2 trials \\
\hline & Reduction of LDL cholesterol levels $(-15 \%)$ in the PLASMA trial in patients with cardiovascular disease[33] \\
\hline & $\begin{array}{l}90 \% \text { reduction in the sPLA2-Ila levels and effective in reducing LDL cholesterol levels and C-reactive protein (CRP) in patients with } \\
\text { stable and acute cardiovascular disease }[23,28]\end{array}$ \\
\hline & Phase 3 trials \\
\hline & $\begin{array}{l}\text { VISTA-16 trial in patients with acute coronary syndrome showed an increased incidence of myocardial infarction and of the total of } \\
\text { cardiovascular events, mortality, heart attack and stroke [36] }\end{array}$ \\
\hline
\end{tabular}

Table 2: Efficacy results of darapladib and varespladib in experimental models and clinical trials

Originally, this enzyme has been described for its activity on PAF (platelet-activating factor) and the capability to hydrolyze oxidized phospholipids (Table 1). Lp-PLA 2 is present mainly in macrophages and in the necrotic core of atherosclerotic lesions of vulnerable plaques $[17,18]$. Regarding its role in atherogenesis, Lp-PLA 2 promotes the accumulation of LDL in the atherosclerotic plaque facilitating their aggregation and oxidation. Unlike sPLA, Lp-PLA is involved in apoptotic cell death of foam cells. The excessive production of lyso-PC by PLA $A_{2}$ could also inhibit the clearance of apoptotic cells perpetuating vascular inflammation and promoting the formation of the necrotic core.

\section{$\mathrm{PLA}_{2}$ as Biomarker of Cardiovascular Risk}

Epidemiological studies conducted during the past decade have documented that plasma levels of both sPLA ${ }_{2}$ and Lp-PLA 2 are correlated with the incidence of cardiovascular disease.

sPLA $_{2}$

Two analyses of the epidemiological study EPIC-Norfolk Prospective Population Study showed a significant association between the activity and the mass of sPLA -IIA and the onset of the first coronary event. This data was then extended to patients with acute coronary syndrome by demonstrating that a high activity of $\mathrm{sPLA}_{2}-$ 
IIA was a predictor factor for further events [19-22]. It should be also noted that a recent meta-analysis, conducted in both the general population and in patients with acute coronary syndrome, showed how the genetic polymorphism in PLA2G2A (rs11573156), associated with reduced mass and activity of $\mathrm{SPLA}_{2}$-IIA, was not associated to major cardiovascular events [23], questioning the potential role of this enzyme in acute coronary syndrome.

\section{PLA $_{2}$ Associated Lipoprotein}

Regarding the Lp-PLA, the first evidence of the link between the enzymatic mass and coronary heart disease derived from the population of the case-control study WOSCOPS [24]. Other studies have confirmed this link in a wide spectrum of populations. The results pf the PEACE trial also demonstrated that high levels of Lp-PLA ${ }_{2}$ are indicators of cardiovascular risk in patients with coronary heart disease, independently of traditional risk factors and hs-CRP [25]. Finally, in patients with stroke, the determination of Lp-PLA ${ }_{2}$ seems to improve the risk stratification [26]. These studies were analyzed in a recent meta-analysis of 32 prospective studies involving 79.036 patients that showed the correlation between activity of Lp-PLA2 and cardiovascular risk [27]. Epidemiological and genetic studies thus represent the most significant evidence of the pro-atherogenicrole of $\mathrm{Lp}^{-\mathrm{LA}_{2}}$ and sPLA IIA.

\section{Pharmacological Inhibitors of Lp-LA ${ }_{2}$ and sPLA}

The aforementioned experimental, epidemiological, and genetic evidences have provided the rationale for the development of varespladib (Anthera) and darapladib (GSK), sPLA and Lp-PLA inhibitors, respectively, in the treatment of cardiovascular diseases [28] (Table 2).

\section{The $\mathrm{SLA}_{2}$ inhibitor varespladib}

Varespladib inhibits human sPLA -IIA, V and $\mathrm{X}$ in a powerful and reversible manner [29] $\left(\mathrm{IC}_{50}=6.2 \mathrm{nM}\right.$ for the IIA) with a selectivity of about 40 times higher than isoform IB [30]. Varespladib was effective in reducing atherosclerosis in murine models of atherosclerosis [31,32], and controlled the development of aneurysm induced by angiotensin II [31]. However, in one of these two studies, varespladib reduced levels of total cholesterol, a possible cause of the observed effects. It is interesting to note that, even in the Phase II PLASMA trial (Phospholipase Levels And Serological Markers of Atherosclerosis), varespladib showed a reduction of cholesterol levels in patients with cardiovascular disease [33]. In guinea pigs, an experimental model characterized by the expression of sPLA ${ }_{2}$-IIA and other isoforms, varespladib treatment did not change the plasma cholesterol levels but reduced the accumulation of lipids in the aortic arch[32]. The observation that varespladib elicits an antiatherosclerotic effect in mice, that do not express the sPLA $A_{2}$-IIA, suggests that the isoforms $\mathrm{PLA}_{2}-\mathrm{V}_{\text {,sPLA }}-\mathrm{X}$, or both contribute to the development of atherosclerosis and are inhibited by this drug.

\section{The Lp-PLA ${ }_{2}$ inhibitor Darapladib}

Darapladib is a potent reversible inhibitor of $\mathrm{Lp}-\mathrm{PLA}_{2}\left(\mathrm{IC}_{50}=\right.$ $0.25 \mathrm{nM}$ ) [28] which causes a significant reduction in the activity of Lp-PLA in atherosclerotic lesions of diabetic/hypercholesterolemic experimental models. Treatment with darapladib reduced the content of lysophosphatidylcholine and attenuated the development of the atherosclerotic plaque [34]. Gene expression analysis has shown an anti-inflammatory effect of darapladib associated with a reduction of the necrotic area [34]. Darapladib has finally demonstrated the ability to reduce the activity of Lp-PLA in human carotid plaques [35].
From these clinical and experimental evidences, it is possible to envision some differences between the two pharmacological approaches. The epidemiological evidences of the role of sPLA $\mathrm{P}_{2}$ on cardiovascular disease are certainly fewer that those provided for the Lp-PLA. Particularly, the validity of a therapeutic approach that inhibits the sPLA -IIA for preventing cardiovascular events has been questioned by the results of a mendelian randomization study [23]. It is also relevant to consider that there are different isoforms ofsPLA ${ }_{2}$ enzymes and only one for the Lp-PLA ${ }_{2}$. Thus, it is likely that a pharmacological agent would not be able to inhibit all the different sPLA $\mathrm{P}_{2}$ isoforms, leading to a possible activation of compensatory sPLA activity that could overcome thepharmacological effect. For these reasons, darapladib could be considered a better pharmacological therapy than varespladib. Nevertheless, the advantage of varespladib in comparison to darapladib is potentially due to its effect on plasma cholesterol levels that could contribute to the eventual antiatherosclerotic properties.

\section{Clinical Trials Conducted with Varespladib and Darapladib}

\section{VISTA-16 trial}

The phase 3 trial VISTA-16 has seen the use of the inhibitor of sPLA varespladib in the treatment of patients with acute coronary syndrome [36].Varespladib not only suppressed the levels of sPLA IIA $(-90 \%)$ but positively affected the lipid-inflammatory status with a significant reduction in the levels of LDL cholesterol and CRP, in patients with both stable and acute cardiovascular (Table 2) $[23,28]$. Patients enrolled in the VISTA- 16 study, treated with $20 \mathrm{mg}$ atorvastatin, were randomized within 96 hours after the coronary event to varespladib (500 $\mathrm{mg}$ daily) or placebo and stratified according to the cholesterol-lowering therapy and the type of event (STEMI, nonSTEMI, unstable angina). The follow-up was 6 months with the visits at $1,2,4,8$ and 16 weeks. The results of the study showed an unfavorable effect of varespladib on cardiovascular events, despite the lower LDL cholesterol levels and CRP compared to placebo. Treatment with varespladib caused an increased incidence of myocardial infarction and events of cardiovascular mortality, heart attack and stroke. These results suggest that, inhibition of $\mathrm{sPLA}_{2}$ in the short term with varespladib is harmful in patients with acute coronary syndrome. One possible explanation of the adverse effects observed could be attributed to the fact that varespladib interferes with the pro-atherosclerotic effects of sPLA 2 -IIA and $\mathrm{V}$ but also with anti-atherosclerotic action of isoform $\mathrm{X}$. Although the precise mechanism behind the increase in the incidence of myocardial infarction has not been elucidated, it is possible that varespladib has induced a pro-thrombotic state, although there was no increase in the post-stent thrombosis events. However, it is important to mention that, other drugs modulating the prostaglandin metabolites have shown a detrimental effect on the incidence of myocardial infarction [37].On the basis of these observations, it is still possible that a selective inhibition of the pro-atherogenicsPLA $\mathrm{P}_{2}$ isoforms can exert a favorable action on atherosclerosis.

\section{Stability trial}

The phase 2 trial IBIS-2, conducted to study the effect of darapladib on plaque stability, showed an arrest in the expansion of the volume of the necrotic core of human carotid plaques assessed by intravascular ultrasound [38]. These findings have led to the hypothesis that darapladib could reduce the risk of cardiovascular events by influencing the composition and the stability of the atherosclerotic plaque. In the phase 3 trial STABILITY (Stabilization of Atherosclerotic Plaque by Darapladib Initiation of Therapy) it has been evaluated the clinical 
efficacy and safety of darapladib in patients with chronic cardiovascular diseases [39]. The study involved the randomization of 15.828 patients to darapladib $160 \mathrm{mg}$ daily or placebo for a period of 3.7 years. All patients were treated according the current guidelines with antiplatelet and statins unless contraindicated or with intolerable side effects. The patients were affected by chronic heart disease, or underwent to a previous myocardial infarction or coronary angioplasty (PCI) or coronary-artery bypass graft $(\mathrm{CABG})$, or had multivascular coronary disease. Darapladib did not significantly reduce the incidence of the primary endpoint, a composite of cardiovascular death, myocardial infarction or stroke. However, there was a significant reduction of the total (a composite of death from coronary heart disease, myocardial infarction, hospitalization for unstable angina, or any coronary revascularization procedure) or major (a composite of death from coronary heart disease, myocardial infarction, or urgent coronary revascularization for myocardial ischemia) coronary events that could be indicative of a possible efficacy of darapladib.

The negative results of this study should be interpreted considering that the patients enrolled were receiving statins, drugs effective in minimizing cardiovascular risk. Indeed, before the randomization, more than a third of the patients had LDL cholesterol levels below $70 \mathrm{mg}$ per deciliter $(1.81 \mathrm{mmol} / \mathrm{L})$ and $75 \%$ underwent to coronary revascularization. Thus, the conventional therapy has certainly reduced the number of events in the two groups of patients, and therefore thosethat were potentially modifiable by administration of darapladib.

A second consideration is related to the fact that $96 \%$ of patients have received, for the entire duration of the study, statin treatment that can reduce the Lp-PLA levels by $35 \%[1,2,9]$. The effect of statins on Lp-PLA 2 is directly related to their ability to reduce the levels of apoB lipoproteins that carry about $70 \%$ of Lp-PLA 2 [29]. Thus, the combination of statins and darapladib could determine an additive cardio protective action through the reduction of Lp-PLA activity.However, the negative results of the study could be explained by at least two reasons: 1)a strong inhibition of Lp-PLA, achieved by the combination of statin and darapladib, does not protect from the cardiovascular death, myocardial infarction, or stroke and thus, the pharmacological intervention on Lp-PLA $A_{2}$ is not an effective therapy for cardiovascular prevention; 2) the effect of darapladib could be masked by the antiatherosclerotic properties of statins or by their anti-inflammatory effects. This second scenario it has been considered relevant also for the development of otherantiathero sclerotic drugs that, for ethical reasons, has been tested in clinical trials in patients already under standard of care treatment.

As previously noted, treatment with darapladib resulted in an increase in the incidence of diarrhea, along the onset of unpleasant odor of the skin, feces and urine, probably caused by the sulfhydryl group of the molecule of darapladib.

In conclusion, the STABILITY trial has evaluated the effectiveness of a new mechanism for reducing the vulnerability of plaque through the inhibition of Lp-PLA with darapladib in patients with chronic cardiovascular disease previously treated with conventional therapies. Darapladib did not significantly reduced the primary endpoint of cardiovascular death, myocardial infarction or stroke [39].

\section{SOLID-TIMI 52 trial}

The therapeutic efficacy of darapladib has also been evaluated in patients with acute coronary syndrome in the phase 3 trial SOLIDTIMI 52 [40]. The study included 13.026 patients, within 30 days of hospitalization for acute coronary syndrome, treated with placebo or darapladib (160 mg daily, 1:1 randomization). Patients were then monitored for an average period of 2.5 years. Similarly to what observed in the STABILITY trial, treatment with darapladib did not alter the primary endpoint of the study of major coronary events (cardiovascular death, myocardial infarction and revascularization). In this case, it has not been observed any favorable effects on secondary endpoints of cardiovascular death, myocardial infarction, stroke, and total mortality. These results were consistent across subgroups including those stratified by baseline LDL cholesterol and the Lp-PLA ${ }_{2}$ activity. Although this study did not demonstrate the efficacy of darapladib, there are several limitations to consider. The study was conducted as a fixed dose of 160 mg darapladib capable to inhibit only $66 \%$ the Lp-PLA activity, without considering doses more intensive. Furthermore, most of cardiovascular events following the acute coronary syndrome were thrombotic and thus not affected by the treatment with darapladib. In conclusion, these results do not justify the use of Lp-PLA, inhibitor darapladib in stable and acute coronary syndrome patients.

\section{Conclusions}

The negative results of the trials VISTA-16, STABILITY and SOLID-TIMI 52, certainly does not put into question the key role of inflammation in atherogenesis, but emphasize the complexity of the pathogenesis of the atherosclerotic process and the need to further investigate the mechanisms underlying the atherogenesis. The interest in this field is also documented by the fact that there are several antiinflammatory drugs currently in clinical development. Novartis has just approved the inhibitor of interleukin 1 (IL-1), canakinumab in a phase 3 study on atherosclerosis and the antisense oligonucleotide ISIS-APOARx directed against the lipoprotein (a) would soon start phase 2. Finally, GSK has initiated a phase 3 trial with the p38 MAPK (mitogen-activated protein kinase) inhibitor, losmapimod, for a shortterm treatment in patients with acute coronary syndrome. Finally, the US National Heart, Lung and Blood Institute have just sponsored a phase 3 trial to evaluate the efficacy of low-dose methotrexate on cardiovascular inflammation. These efforts will help to clarify the efficacy of therapeutic agents with vascular anti-inflammatory activity on the control of atherosclerosis and cardiovascular diseases.

\section{References}

1. [No authors listed] (1994) Randomised trial of cholesterol lowering in 4444 patients with coronary heart disease: the Scandinavian Simvastatin Survival Study (4S) Lancet 344: 1383-1389

2. LaRosa JC, Grundy SM, Waters DD, Shear C, Barter P, et al. (2005) Intensive lipid lowering with atorvastatin in patients with stable coronary disease. $\mathrm{N}$ Engl J Med 352: 1425-1435.

3. Ridker PM, Danielson E, Fonseca FA, Genest J, Gotto AM Jr, et al. (2009) Reduction in C-reactive protein and LDL cholesterol and cardiovascular event rates after initiation of rosuvastatin: a prospective study of the JUPITER trial Lancet373: 1175-1182.

4. Bellosta S, Ferri N, Arnaboldi L, Bernini F, Paoletti R, et al. (2000) Pleiotropic effects of statins in atherosclerosis and diabetes. Diabetes Care 23 Suppl 2: B72-78.

5. Bellosta S, Ferri N, Bernini F, Paoletti R, Corsini A (2000) Non-lipid-related effects of statins. Ann Med 32: 164-176.

6. Corsini A, Ferri N, Cortellaro M (2007) Are pleiotropic effects of statins real? Vasc Health Risk Manag 3: 611-613.

7. Ferri N, Corsini A2 (2014) Clinical evidence of statin therapy in non-dyslipidemic disorders. Pharmacol Res 88: 20-30.

8. Ferri N, Paoletti R, Corsini A (2005) Lipid-modified proteins as biomarkers for cardiovascular disease: a review. Biomarkers 10:219-237.

9. Mallat Z, Lambeau G, Tedgui A (2010) Lipoprotein-associated and secreted phospholipases $A_{2}$ in cardiovascular disease: roles as biological effectors and biomarkers. Circulation 122: 2183-2200. 
Citation: Ferri N, Ricci C, Corsini A (2015) Pharmacological Inhibition of Phosholipase A2: Results from Phase 3 Clinical Trials with Darapladib and Varespladib in Patients with Cardiovascular Disease. Cardiol Pharmacol 4: 137. doi:10.4172/2329-6607.1000137

Page 5 of 5

10. Elinder LS, Dumitrescu A, Larsson P, Hedin U, Frostegård J, et al. (1997) Expression of phospholipase A2 isoforms in human normal and atherosclerotic arterial wall. Arterioscler Thromb Vasc Biol 17: 2257-2263.

11. Menschikowski M, Kasper M, Lattke P, Schiering A, Schiefer S, et al. (1995) Secretory group II phospholipase A2 in human atherosclerotic plaques. Atherosclerosis 118: 173-181.

12. Rosengren B, Jonsson-Rylander AC, Peilot H, Camejo G, Hurt-Camejo E (2006) Distinctiveness of secretory phospholipase A2 group IIA and V suggesting unique roles in atherosclerosis. BiochimBiophysActa1761: 13011308.

13. Rosengren $B$, Peilot $H$, Umaerus $M$, Jönsson-Rylander AC, Mattsson-Hultén $L$, et al. (2006) Secretory phospholipase A2 group V: lesion distribution, activation by arterial proteoglycans, and induction in aorta by a Western diet. Arterioscler Thromb Vasc Biol 26: 1579-1585.

14. Vickers KC, Maguire CT, Wolfert R, Burns AR, Reardon M, et al. (2009) Relationship of lipoprotein-associated phospholipase A2 and oxidized low density lipoprotein in carotid atherosclerosis. J Lipid Res 50: 1735-1743.

15. Karabina SAI, Brocheriou G, Le Naour, Agrapart M, Durand H, et al. (2006) Atherogenic properties of LDL particles modified by human group $X$ secreted phospholipase A2 on human endothelial cell function. FASEB J 20: 2547-2549.

16. Kimura-Matsumoto M, IshikawaY, Komiyama K,Tsurutab T, MurakamiM, et al (2008) Expression of secretory phospholipase A2s in human atherosclerosis development. Atherosclerosis 196: 81-91.

17. Kolodgie FD, Burke AP, Skorija KS, Kutys R, Makuria AT, et al. (2006) Lipoprotein-associated phospholipase A2 protein expression in the natura progression of human coronary atherosclerosis. Arterioscler Thromb Vasc Biol 26: $2523-2529$

18. Lavi S, McConnell JP, Rihal CS, Prasad A, Mathew V, et al. (2007) Local production of lipoprotein-associated phospholipase A2 and lysophosphatidylcholine in the coronary circulation: association with early coronary atherosclerosis and endothelial dysfunction in humans. Circulation 115: $2715-2721$

19. Boekholdt SM, Keller TT, Wareham NJ, Luben R, Bingham SA, et al. (2005) Serum levels of type II secretory phospholipase A2 and the risk of future coronary artery disease in apparently healthy men and women: the EPICNorfolk Prospective Population Study. Arterioscler Thromb Vasc Biol 25: 839-

20. Kugiyama K, Ota Y, Takazoe K, Moriyama Y, Kawano H, et al. (1999) Circulating levels of secretory type II phospholipase $A(2)$ predict coronary events in patients with coronary artery disease. Circulation 100: 1280-1284.

21. Mallat Z, Benessiano J, Simon T, Ederhy S, Sebella-Arguelles C, et al. (2007) Circulating secretory phospholipase A2 activity and risk of incident coronary events in healthy men and women: the EPIC-Norfolk study. Arterioscler Thromb Vasc Biol 27: 1177-1183.

22. Mallat Z, Steg PG, Benessiano J, Tanguy ML, Fox KA, et al. (2005) Circulating secretory phospholipase A2 activity predicts recurrent events in patients with severe acute coronary syndromes. J Am Coll Cardiol 46: 1249-1257.

23. Holmes MV, Simon T, Exeter HJ, Folkersen L, Asselbergs FW, et al. (2013) Secretory phospholipase $\mathrm{A}(2)-\mathrm{II}$ a and cardiovascular disease: a mendelian randomization study. J Am Coll Cardiol 62: 1966-1976.

24. Packard CJ, O'Reilly DS, Caslake MJ, McMahon AD, Ford I, et al. (2000) Lipoprotein-associated phospholipase A2 as an independent predictor of coronary heart disease. West of Scotland Coronary Prevention Study Group. N Engl J Med 343:1148-1155.

25. Sabatine MS, Morrow DA, O'Donoghue M, Jablonksi KA, Rice MM, et al. (2007) Prognostic utility of lipoprotein-associated phospholipase A2 for cardiovascular outcomes in patients with stable coronary artery disease. Arterioscler Thromb Vasc Biol 27: 2463-2469.

26. Nambi V,Hoogeveen RC, ChamblessL, Hu Y, Bang H, et al.(2009) Lipoproteinassociated phospholipase $\mathrm{A} 2$ and high-sensitivity $\mathrm{C}$-reactive protein improve the stratification of ischemic stroke risk in the Atherosclerosis Risk in Communities (ARIC) study. Stroke 40: 376-381.

27. The Lp-PLA Studies Collaboration (2010) Lipoprotein-associated phospholipase $\mathrm{A}(2)$ and risk of coronary disease, stroke, and mortality: collaborative analysis of 32 prospective studies. Lancet 375: 1536-1544

28. Blackie JA, Bloomer JC, Brown MJ, Cheng HY, Hammond B, et al. (2003) The identification of clinical candidate SB-480848: a potent inhibitor of lipoprotein- associated phospholipase A2. Bioorg Med ChemLett 13: 1067-1070.

29. [No authors listed] (2011) Varespladib. Am J Cardiovasc Drugs 11: 137-143.

30. Snyder DW, Bach NJ, Dillard RD, Draheim SE, Carlson DG, et al. (1999) Pharmacology of LY315920/S-5920, [[3-(aminooxoacetyl)-2-ethyl-1(phenylmethyl)-1H-indol-4-yl]oxy] acetate, a potent and selective secretory phospholipase A2 inhibitor: A new class of anti-inflammatory drugs, SPI. J PharmacolExpTher288: 1117-1124.

31. Fraser H, Hislop C, Christie RM, Rick HL, Reidy CA, et al. (2009) Varespladib (A-002), a secretory phospholipase A2 inhibitor, reduces atherosclerosis and aneurysm formation in ApoE-/- mice. J Cardiovasc Pharmacol 53: 60-65.

32. Shaposhnik Z, Wang X, Trias J, Fraser H, Lusis AJ (2009) The synergistic inhibition of atherogenesis in apoE-/- mice between pravastatin and the SPLA2 inhibitor varespladib (A-002). J Lipid Res 50: 623-629.

33. Rosenson RS (2009) Future role for selective phospholipase A2 inhibitors in the prevention of atherosclerotic cardiovascular disease. Cardiovasc Drugs Ther 23: 93-101.

34. Wilensky RL, Shi Y, Mohler ER 3rd, Hamamdzic D, Burgert ME, et al. (2008) Inhibition of lipoprotein-associated phospholipase A2 reduces complex coronary atherosclerotic plaque development. Nat Med 14: 1059-1066.

35. Johnson JL, Shi Y, Snipes R, Janmohamed S, Rolfe TE, et al. (2014) Effect of darapladib treatment on endarterectomy carotid plaque lipoprotein-associated phospholipase A2 activity: a randomized, controlled trial. PLoS One 9: e89034.

36. Nicholls SJ, Kastelein JJ, Schwartz GG, Bash D, Rosenson RS, et al. (2014) Varespladib and cardiovascular events in patients with an acute coronary syndrome: the VISTA-16 randomized clinical trial. JAMA 311: 252-262.

37. Mohler ER 3rd, Ballantyne CM, Davidson MH, Hanefeld M, Ruilope LM, et al. (2008) The effect of darapladib on plasma lipoprotein-associated phospholipase A2 activity and cardiovascular biomarkers in patients with stable coronary hear disease or coronary heart disease risk equivalent: the results of a multicenter randomized, double-blind, placebo-controlled study. J Am Coll Cardiol 51: 1632-1641.

38. Serruys PW, Garcia-Garcia HM, Buszman P, Erne P, Verheye S, et al. (2008) Effects of the direct lipoprotein-associated phospholipase $A(2)$ inhibitor darapladib on human coronary atherosclerotic plaque. Circulation 118: 11721182.

39. STABILITY Investigators, White HD, Held C, Stewart R, Tarka E, et al. (2014) Darapladib for preventing ischemic events in stable coronary heart disease. $\mathrm{N}$ Engl J Med 370: 1702-1711.

40. O'Donoghue ML, Braunwald E, White HD, Lukas MA, Tarka E, et al. (2014) Effect of darapladib on major coronary events after an acute coronary syndrome: the SOLID-TIMI 52 randomized clinical trial. JAMA 312: 1006-1015. 